\title{
DIRC3 and near NABP1 genetic polymorphisms are associated laryngeal squamous cell carcinoma patient survival
}

\author{
Zhen Shen ${ }^{1, *}$, Wanli Ren ${ }^{1, *}$, Yanxia Bai ${ }^{1}$, Zhengshuai Chen ${ }^{3,4}$, Jingjie Li ${ }^{3,4}$, Bin $\mathrm{Li}^{3,4}$, \\ Tianbo Jin ${ }^{3,4}$, Peilong Cao', Yuan Shao ${ }^{1}$ \\ ${ }^{1}$ Department of Otolaryngology \& head neck, The First Affiliated Hospital of Xi'an Jiaotong University, Xi'an, Shaanxi 710061, \\ China \\ ${ }^{2}$ Department of Pathology, The First Affiliated Hospital of Xi'an Jiaotong University, Xi'an, Shaanxi 710061, China \\ ${ }^{3}$ School of Life Sciences, Northwest University, Xi'an, Shaanxi 710069, China \\ ${ }^{4}$ National Engineering Research Center for Miniaturized Detection Systems, Xi'an 710069, China \\ *These authors have contributed equally to this work \\ Correspondence to: Peilong Cao, email: peilonggao@163.com \\ Yuan Shao, email: jiaodashaoyuan@163.com
}

Keywords: laryngeal squamous cell carcinoma, NABP 1, DIRC3, biomarker, polymorphism

Received: August 11,2016 Accepted: October 14,2016 Published: October 25, 2016

\section{ABSTRACT}

Laryngeal squamous cell carcinoma (LSCC) is one of the most common and aggressive malignancies of the upper digestive tract. The present study is a retrospective analysis of data from a prospective longitudinal study. A total of 170 male LSCC patients (average age, 60.75 10.082) at the First Affiliated Hospital of Xi'an Jiaotong University School of Medicine were recruited between January 2002 and April 2013 for this study. We assessed correlations between patient characteristics and survival, and sequenced genomic DNA from patient peripheral blood samples. We found that the single nucleotide polymorphisms (SNPs), rs11903757, with closest proximity to NABP1 and SDPR, and rs966423 in DIRC3, were associated with survival in LSCC patients. Median follow-up was 38 months (range 3-122) and median survival time was 48 months. LSCC patients with total laryngectomy, poor differentiation, T3T4 stage, N1-N2 stage or III-IV TNM stage had reduced survival. This is the first study to demonstrate that the rs11903757 GT $(\mathrm{HR}=2.036 ; 95 \% \mathrm{CI}, 1.071-3.872 ; p=0.030)$ and rs966423 TT (HR=11.677; 95\% CI, 3.901-34.950; $p=0.000)$ genotypes predict poor patient outcome. These polymorphisms may serve as useful clinical markers to predict patient survival, and to guide individual patient therapeutic decisions.

\section{INTRODUCTION}

Carcinomas of the upper aerodigestive tract represent a major challenge in modern health care. Laryngeal squamous cell carcinoma (LSCC), which directly impacts patient speech and communication, is one of the most common and aggressive malignancies of the upper digestive tract $[1,2]$. For LSCC patients, poor early diagnosis rates result in delayed treatment and higher levels of disease recurrence and metastasis.

In China, LSCC incidence has been rising gradually, especially in the northeast. Despite significant advances in surgery and radio-therapeutic techniques and new chemotherapeutics, the 5-year relative survival rate for LSCC patients has not markedly improved, and mortality is still high at 1.2 cases per 100,000 persons [6]. Additionally, there are currently no ideal prognostic biomarkers to guide laryngeal cancer patient treatment. Presently, much work is focused on the identification of useful diagnostic and therapeutic markers [7, 8], and recent findings suggest that the combined expression patterns of multiple genes may be useful prognostic indicators. Tissue microarray and proteomics technologies have enabled the discovery of LSCC metastasis suppressor genes, which have strong potential for use as biomarkers. Still, high morbidity and low cure rates necessitate the development of new and improved diagnostic procedures, biomarkers and therapeutics to effectively treat LSCC [3-5]. 
The present study is a retrospective analysis using data from a prospective longitudinal study of 170 patients over an extended time period (2002-2013). We examined LSCC patient epidemiology with regard to age, laryngectomy, neck dissection, tumor differentiation, T-stage, N-stage, TNM stage and treatment modality. In analyzing possible genetic polymorphisms associated with LSCC susceptibility and prognosisS, we hoped to identify possible diagnostic markers or therapeutic targets that could lead to improved patient survival.

\section{RESULTS}

\section{Patient characteristics and treatment outcomes}

A total of 170 LSCC patients at the First Affiliated Hospital of Xi' an Jiaotong University School of Medicine were recruited from January 2002 and April 2013 for this study. All patients were males with an average age of $60.75 \pm 10.082$ years (mean $\pm \mathrm{SD}$ ) (range, 32-82 years). Patients were followed up from diagnosis until the end of April, 2013. No patient was lost from follow-up data. The median follow-up time was 38 months (range, 3-122 months) and median survival time was 48 months. $100 / 170(58.82 \%)$ patients died of LSCC during the study period. The 5 -year overall survival rate was $47.1 \%$ (Figure 1A). Patient deaths were regarded as censored data and were so marked on survival curves. Characteristics of the 14 candidate single nucleotide polymorphisms (SNPs) assessed in this study are shown in Table 1.

Potential factors that influenced the prognosis are shown in Table 2. We investigated relationships between these factors and survival by univariate analysis, and survival curves were drawn using the Kaplan-Meier method. We found that patient laryngectomy ( $p=0.000$, Figure $1 \mathrm{~B})$, differentiation $(p=0.008$, Figure $1 \mathrm{C})$, T-stage $(p=0.000$, Figure 1D), N-stage ( $p=0.000$, Figure 1E), and TNM stage ( $p=0.000$, Figure $1 \mathrm{~F}$ ) were associated with survival based on the log-rank test. However, survival was not correlated with age $(p=0.456)$ and neck dissection $(p=0.188)$ in stratified analyses. Compared with partial laryngectomy, high differentiation, T1-T2 stage, N0 stage and I-II TNM stage, the HR for total laryngectomy (95\% CI, 1.576-3.492; $p=0.000$ ), poor differentiation (95\% CI, 1.150-4.997; $p=0.020)$, T3-T4 stage (95\% CI, $1.448-3.253 ; p=0.000)$, N1N2 stage $(95 \% \mathrm{CI}, 1.582-3.623 ; p=0.000)$ and III-IV TNM stage (95\% CI, 2.100-5.180; $p=0.000)$ increased to 2.346 , $2.397,2.170,2.394$ and 3.298, respectively.

\section{Genetic polymorphisms and outcome correlations}

We assessed the associations between 4 genotypes and survival by univariate analysis (Table 3 ). Two SNPs
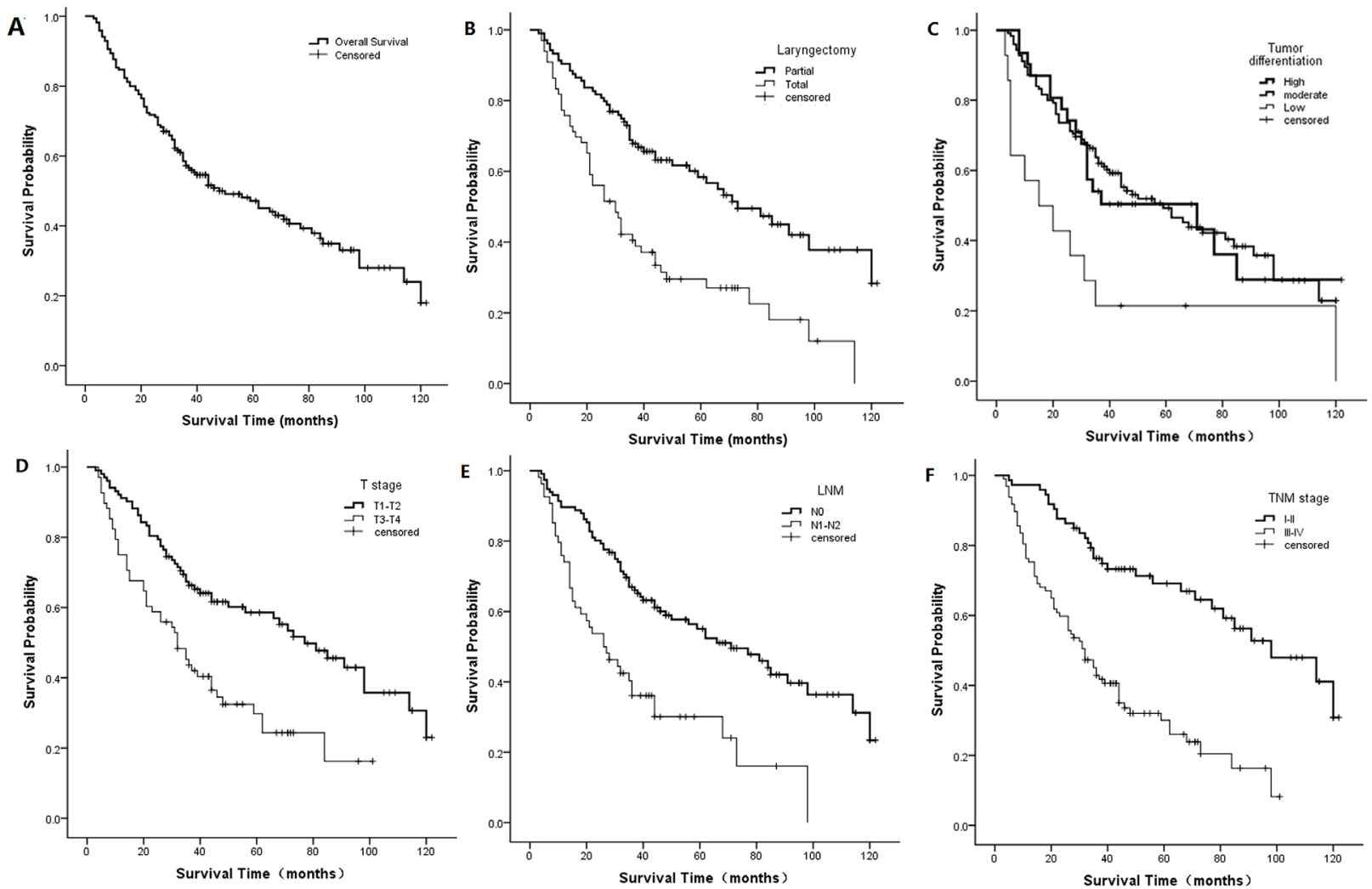

Figure 1: Kaplan-Meier curves for potential factors influencing LSCC patient survival. Overall survival A. laryngectomy $(p=0.000, \log$-rank test) B. Tumor differentiation $(p=0.008, \log$-rank test $)$ C. T-stage $(p=0.000, \log$-rank test $) \mathbf{D} . \mathrm{N}$-stage $(p=0.000, \log$-rank test) E. TNM stage ( $p=0.000$, log-rank test) F. Curves represent 10 years of follow-up. 
Table 1: Basic information of SNPs in this study

\begin{tabular}{lccccc}
\hline SNP-ID & Position & Band & Allele & Gene & Role \\
\hline rs17401966 & 10385471 & $1 \mathrm{p} 36.22$ & $\mathrm{G} / \mathrm{A}$ & $\mathrm{KIF} 1 \mathrm{~B}$ & Intron \\
rs4072037 & 155162067 & $1 \mathrm{q} 22$ & $\mathrm{G} / \mathrm{A}$ & $\mathrm{MUC1}$ & Coding exon \\
rs1912453 & 162821291 & $1 \mathrm{q} 23.3$ & $\mathrm{C} / \mathrm{T}$ & $\mathrm{C} 1 \mathrm{orf110}$ & Downstream \\
rs10911251 & 183081194 & $1 \mathrm{q} 25.3$ & $\mathrm{~A} / \mathrm{C}$ & $\mathrm{LAMC1}$ & Intron \\
rs6687758 & 222164948 & $1 \mathrm{q} 41$ & $\mathrm{G} / \mathrm{A}$ & - & - \\
rs11903757 & 192587204 & $2 \mathrm{q} 32.3$ & $\mathrm{C} / \mathrm{T}$ & - & - \\
rs9288520 & 217481271 & $2 \mathrm{q} 35$ & $\mathrm{~A} / \mathrm{G}$ & - & - \\
rs966423 & 218310340 & $2 \mathrm{q} 35$ & $\mathrm{~T} / \mathrm{C}$ & $\mathrm{DIRC} 3$ & Intron \\
rs6736997 & 235615197 & $2 \mathrm{q} 37.2$ & $\mathrm{~A} / \mathrm{C}$ & & - \\
rs975334 & 2846316 & $3 \mathrm{p} 26.2$ & $\mathrm{C} / \mathrm{T}$ & $\mathrm{CNTN4}$ & Intron \\
rs8180040 & 47388947 & $3 \mathrm{p} 21.31$ & $\mathrm{~A} / \mathrm{T}$ & KLHL18 & Downstream \\
rs9841504 & 114362764 & $3 \mathrm{q} 13.31$ & $\mathrm{G} / \mathrm{C}$ & ZBTB20 & Intron \\
rs10936599 & 169492101 & $3 \mathrm{q} 26.2$ & $\mathrm{~T} / \mathrm{C}$ & $\mathrm{ARPM1}$ & Promoter \\
rs2239612 & 186793242 & $3 \mathrm{q} 27.3$ & $\mathrm{~T} / \mathrm{C}$ & $\mathrm{ST6GAL1}$ & Intron \\
\hline
\end{tabular}

Table 2: Patient characteristics and treatment outcomes - univariate associations with LSCC prognosis

\begin{tabular}{|c|c|c|c|c|c|}
\hline & N (Total/Events) & Median & $P$-value ${ }^{a}$ & HR $(95 \%$ CI) & $P$-value ${ }^{b}$ \\
\hline \multicolumn{6}{|l|}{ Age (years) } \\
\hline$<60$ & $80 / 47$ & 59 & \multirow{2}{*}{0.456} & 1 & \\
\hline$\geq 60$ & $90 / 53$ & 48 & & $1.161(0.782-1.722)$ & 0.460 \\
\hline \multicolumn{6}{|c|}{ Laryngectomy } \\
\hline Partial & $104 / 50$ & 73 & \multirow{2}{*}{$0.000 *$} & 1 & \\
\hline Total & $66 / 50$ & 30 & & $2.346(1.576-3.492)$ & $0.000 *$ \\
\hline \multicolumn{6}{|c|}{ Neck dissection } \\
\hline yes & $37 / 19$ & 36 & \multirow{2}{*}{0.188} & 1 & \\
\hline no & $133 / 81$ & 56 & & $0.711(0.42-1.189)$ & 0.194 \\
\hline \multicolumn{6}{|c|}{ Differentiation } \\
\hline high & $31 / 18$ & 71 & \multirow{3}{*}{$0.008^{*}$} & 1 & \\
\hline moderate & $125 / 70$ & 59 & & $0.933(0.556-1.567)$ & 0.794 \\
\hline poor & $14 / 12$ & 15 & & $2.397(1.150-4.997)$ & $0.020 *$ \\
\hline \multicolumn{6}{|l|}{ T-stage } \\
\hline $\mathrm{T} 1-\mathrm{T} 2$ & $102 / 52$ & 77 & \multirow{2}{*}{$0.000 *$} & 1 & \\
\hline T3-T4 & $68 / 48$ & 32 & & $2.170(1.448-3.253)$ & $0.000^{*}$ \\
\hline \multicolumn{6}{|l|}{ N-stage } \\
\hline N0 & $116 / 61$ & 71 & \multirow{2}{*}{$0.000 *$} & 1 & \\
\hline N1-N2 & $54 / 39$ & 26 & & $2.394(1.582-3.623)$ & $0.000 *$ \\
\hline \multicolumn{6}{|l|}{ TNM stage } \\
\hline I-II & $73 / 30$ & 98 & \multirow{2}{*}{$0.000 *$} & 1 & \\
\hline III, IV & $97 / 70$ & 32 & & $3.298(2.100-5.180)$ & $0.000 *$ \\
\hline
\end{tabular}

Notes: ${ }^{\mathrm{a}} P$-values based on the log-rank test; ${ }^{\mathrm{b}} P$-values based on the Wald test; ${ }^{*} \mathrm{p}$-value $<0.05$ indicates statistical significance.

Abbreviations: HR, hazard ratios; CI: confidence intervals. 
Table 3: Genetic polymorphisms and outcome - univariate associations with LSCC prognosis

\begin{tabular}{|c|c|c|c|c|c|c|}
\hline SNP-ID & genotype & N(Total/Events) & Median & $P$-value ${ }^{a}$ & HR (95\% CI) & $P$-value ${ }^{b}$ \\
\hline \multirow[t]{3}{*}{ rs17401966 } & $\mathrm{A} / \mathrm{A}$ & $125 / 73$ & 48.0 & & 1 & \\
\hline & $\mathrm{G} / \mathrm{A}$ & $36 / 19$ & 66.0 & 0.437 & $0.905(0.545-1.502)$ & 0.669 \\
\hline & $\mathrm{G} / \mathrm{G}$ & $8 / 7$ & 35.0 & & $1.576(0.724-3.430)$ & 0.252 \\
\hline \multirow[t]{3}{*}{ rs4072037 } & $\mathrm{A} / \mathrm{A}$ & 16394 & 46 & & 1 & \\
\hline & $\mathrm{G} / \mathrm{A}$ & $2 / 2$ & 81 & 0.843 & $0.976(0.239-3.985)$ & 0.973 \\
\hline & $\mathrm{G} / \mathrm{G}$ & $3 / 3$ & 59 & & $1.403(0.442-4.450)$ & 0.565 \\
\hline \multirow[t]{3}{*}{ rs 1912453} & $\mathrm{~T} / \mathrm{T}$ & $41 / 25$ & 46 & & 1 & \\
\hline & $\mathrm{C} / \mathrm{T}$ & $113 / 65$ & 62 & 0.389 & $0.936(0.589-1.486)$ & 0.779 \\
\hline & $\mathrm{C} / \mathrm{C}$ & $15 / 10$ & 35 & & $1.489(0.712-3.116)$ & 0.290 \\
\hline \multirow[t]{3}{*}{ rs10911251 } & $\mathrm{C} / \mathrm{C}$ & $45 / 28$ & 36 & & 1 & \\
\hline & $\mathrm{A} / \mathrm{C}$ & $81 / 48$ & 59 & 0.720 & $0.831(0.518-1.333)$ & 0.443 \\
\hline & $\mathrm{A} / \mathrm{A}$ & $34 / 19$ & 66 & & $0.949(0.530-1.700)$ & 0.860 \\
\hline \multirow[t]{3}{*}{ rs6687758 } & $\mathrm{A} / \mathrm{A}$ & $114 / 68$ & 59 & & 1 & \\
\hline & $\mathrm{G} / \mathrm{A}$ & $44 / 26$ & 44 & 0.979 & $1.047(0.664-1.651)$ & 0.842 \\
\hline & $\mathrm{G} / \mathrm{G}$ & $5 / 2$ & 34 & & $0.975(0.238-4.000)$ & 0.972 \\
\hline \multirow[t]{3}{*}{ rs11903757 } & $\mathrm{T} / \mathrm{T}$ & $156 / 88$ & 59 & & 1 & \\
\hline & $\mathrm{C} / \mathrm{T}$ & $14 / 12$ & 23 & $0.021^{*}$ & $2.001(1.091-3.673)$ & $0.025 *$ \\
\hline & $\mathrm{C} / \mathrm{C}$ & $-/-$ & - & & - & - \\
\hline \multirow[t]{3}{*}{ rs9288520 } & $\mathrm{G} / \mathrm{G}$ & $92 / 52$ & 48 & & 1 & \\
\hline & $\mathrm{A} / \mathrm{G}$ & $72 / 45$ & 50 & 0.786 & $1.085(0.727-1.619)$ & 0.691 \\
\hline & $\mathrm{A} / \mathrm{A}$ & $4 / 2$ & 22 & & $0.692(0.166-2.877)$ & 0.613 \\
\hline \multirow[t]{3}{*}{ rs966423 } & $\mathrm{C} / \mathrm{C}$ & $111 / 65$ & 62 & & 1 & \\
\hline & $\mathrm{T} / \mathrm{C}$ & $52 / 29$ & 44 & $0.000 *$ & $1.089(0.701-1.690)$ & 0.705 \\
\hline & $\mathrm{T} / \mathrm{T}$ & $4 / 4$ & 5 & & $7.721(2.748-21.695)$ & $0.000^{*}$ \\
\hline \multirow[t]{3}{*}{ rs6736997 } & $\mathrm{C} / \mathrm{C}$ & $105 / 66$ & 44 & & 1 & \\
\hline & $\mathrm{A} / \mathrm{C}$ & $64 / 33$ & 62 & 0.540 & $0.878(0.577-1.336)$ & 0.543 \\
\hline & $\mathrm{A} / \mathrm{A}$ & $-/-$ & - & & - & - \\
\hline \multirow[t]{3}{*}{ rs975334 } & $\mathrm{T} / \mathrm{T}$ & $125 / 74$ & 48 & & 1 & \\
\hline & $\mathrm{C} / \mathrm{T}$ & $42 / 25$ & 46 & 0.584 & $1.017(1.017-1.603)$ & 0.941 \\
\hline & $\mathrm{C} / \mathrm{C}$ & $3 / 1$ & 59 & & $0.373(0.052-2.687)$ & 0.328 \\
\hline \multirow[t]{3}{*}{ rs8180040 } & $\mathrm{T} / \mathrm{T}$ & $51 / 32$ & 44 & & 1 & \\
\hline & $\mathrm{A} / \mathrm{T}$ & $51 / 28$ & 62 & 0.754 & $0.836(0.501-1.394)$ & 0.493 \\
\hline & $\mathrm{A} / \mathrm{A}$ & $43 / 24$ & 62 & & $0.858(0.505-1.458)$ & 0.572 \\
\hline \multirow[t]{3}{*}{ rs9841504 } & $\mathrm{G} / \mathrm{G}$ & $132 / 77$ & 56 & & 1 & \\
\hline & $\mathrm{G} / \mathrm{C}$ & $26 / 16$ & 40 & 0.680 & $1.120(0.652-1.925)$ & 0.682 \\
\hline & $\mathrm{C} / \mathrm{C}$ & $3 / 2$ & 26 & & $1.763(0.430-7.223)$ & 0.431 \\
\hline \multirow[t]{3}{*}{ rs10936599 } & $\mathrm{C} / \mathrm{C}$ & $48 / 34$ & 44 & & 1 & \\
\hline & $\mathrm{T} / \mathrm{C}$ & $73 / 37$ & 62 & 0.153 & $0.635(0.398-1.014)$ & 0.057 \\
\hline & $\mathrm{C} / \mathrm{C}$ & $47 / 28$ & 36 & & $0.818(0.494-1.356)$ & 0.436 \\
\hline \multirow[t]{3}{*}{ rs2239612 } & $\mathrm{C} / \mathrm{C}$ & $101 / 54$ & 71 & & 1 & \\
\hline & $\mathrm{T} / \mathrm{C}$ & $45 / 31$ & 40 & 0.192 & $1.488(0.954-2.321)$ & 0.080 \\
\hline & $\mathrm{T} / \mathrm{T}$ & $12 / 8$ & 44 & & $1.332(0.632-2.810)$ & 0.451 \\
\hline
\end{tabular}

Notes: ${ }^{a} P$-values based on the log-rank test; ${ }^{b} P$-values based on the Wald test; $*$ p-value $<0.05$ indicates statistical significance.

Abbreviations: HR, hazard ratios; CI: confidence intervals. 
(rs11903757, $p=0.021$, Figure 2A; rs966423, $p=0.000$, Figure 2B) were associated with survival when all patients were examined using the log-rank test. For rs11903757 near NABP1, the CT genotype (HR, 2.001; $95 \% \mathrm{CI}, 1.091-3.673 ; p=0.025)$ resulted in a higher risk than the TT genotype. With regard to rs966423 in DIRC3, survival was worse in patients with the TT genotype (HR, 7.721; 95\% CI, 2.748-21.695; $p=0.000$ ) compared to those with the CC genotype. The TC genotype (HR, 1.089; 95\% CI, 0.701-1.690; $p=0.705$ ) was not associated with survival.
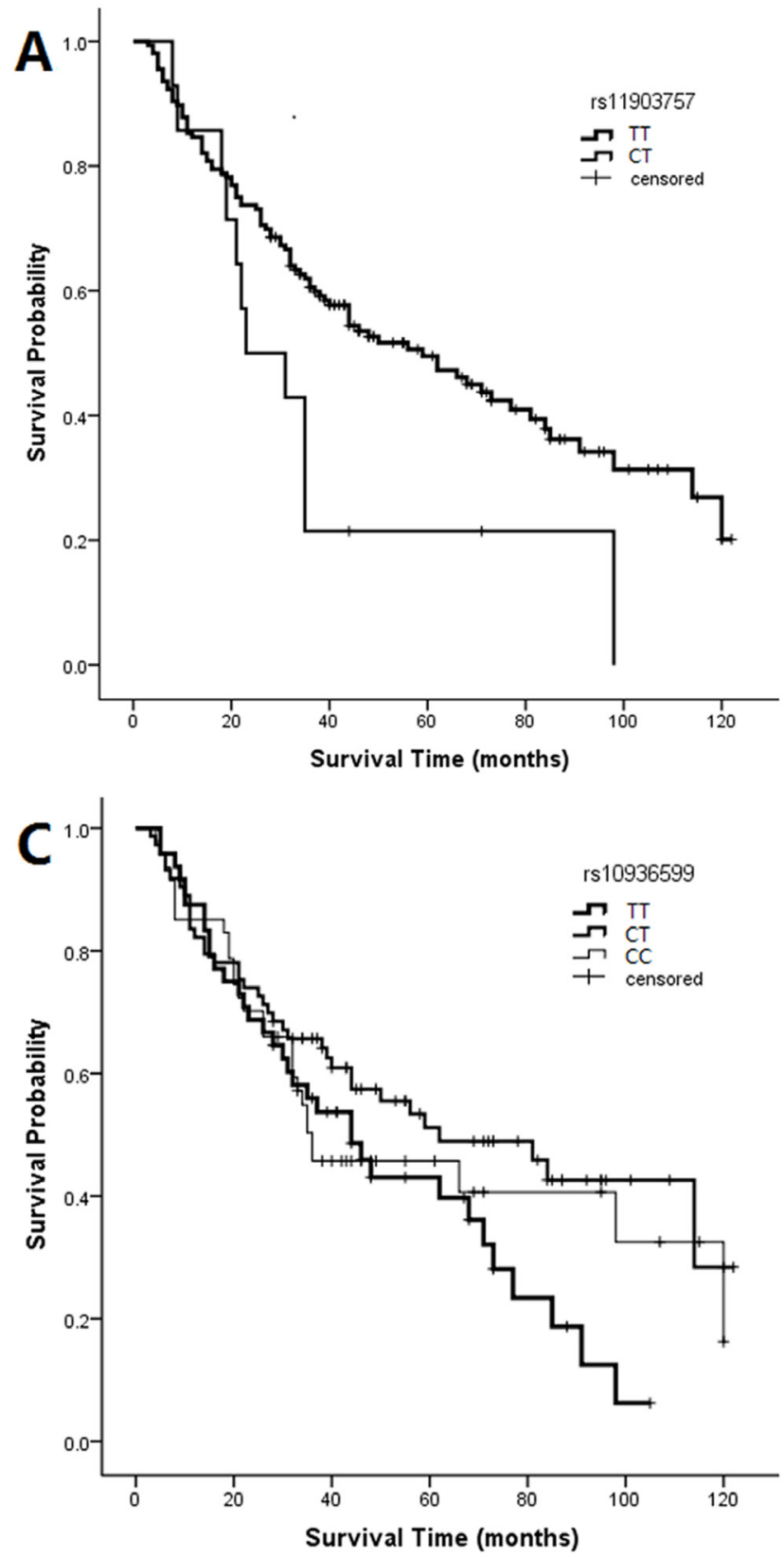

\section{Multivariate analysis of treatment outcome}

We hypothesized that the rs11903757 and rs966423 genotypes might be prognostic markers in LSCC. Univariate analysis showed that patients with total laryngectomy, poor differentiation, T3-T4 stage, N1N2 stage, III-IV TNM stage or these SNPs had worse survival. This suggests that rs10936599 $(p=0.153$, Figure 2C) in the promoter region of ARPM1 and rs2239612 ( $p=0.192$, Figure 2D) in the intron region of ST6GAL1 might be associated with prognostic survival. We adjusted for the above confounding factors in a multivariate Cox
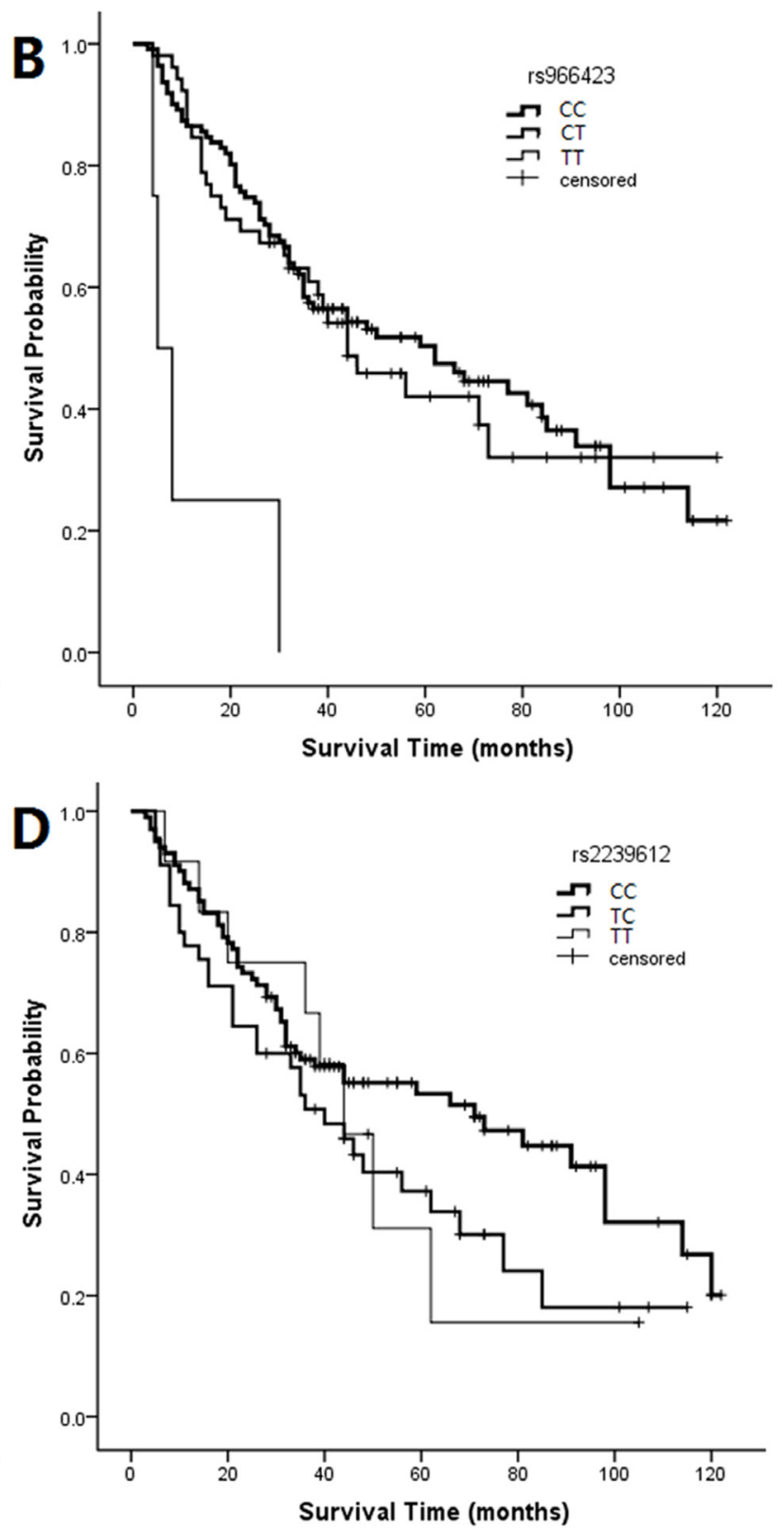

Figure 2: Kaplan-Meier curves for SNPs associated with LSCC patient survival. rs 11903757 genotypes $(p=0.021$, log-rank test) A. rs 966423 genotypes $(p=0.000$, log-rank test) B. rs 10936599 genotypes ( $p=0.153$, log-rank test) C. $r$ 2239612 genotypes $(p=0.192$, log-rank test) D. Graphs represent 10 years of follow-up. 
Table 4: Genetic polymorphisms and outcome - multivariate associations with LSCC prognosis

\begin{tabular}{lccc}
\hline SNP-ID & Genotype & HR(95\% CI) & P-value \\
\hline rs11903757 & TT & 1 & $\mathbf{0 . 0 3 0 *}$ \\
rs966423 & CT & $2.036(1.071-3.872)$ & 0.543 \\
rs 10936599 & CC & 1 & $\mathbf{0 . 0 0 0 *}$ \\
TC 2239612 & TT & $0.154(0.727-1.833)$ & $11.677(3.901-34.950)$ \\
TT & CT & $0.780(0.475-1.280)$ & 0.326 \\
CC & CC & $0.933(0.555-1.567)$ & 0.792 \\
& TC & $1.232(0.779-1.949)$ & 0.373 \\
\hline
\end{tabular}

Notes: ${ }^{\mathrm{b}} P$-values based on the Wald test; ${ }^{*}$ p-value $<0.05$ indicates statistical significance.

Abbreviations: HR, hazard ratios; CI: confidence intervals.

proportional hazards model, calculating $\mathrm{HR}$ and $95 \% \mathrm{CI}$ for different genotypes, to measure the impacts of alleles on prognosis (Table 4). With regard to rs 11903757 , the genotype CT (HR, 2.036; 95\% CI, 1.071-3.872; $p=0.030$ ) increased the HR more than two-fold compared to the TT genotype. For rs966423, survival was worse in patients with the TT genotype (HR, 11.677; 95\% CI, 3.901-34.950; $p=0.000$ ) than those with the CC genotype. Rs10936599 and rs2239612 were not related to prognosis.

\section{DISCUSSION}

Laryngeal cancer is the second most common type of head and neck cancer. An estimated 12,000 new laryngeal cancer cases are diagnosed in the USA every year. The incidence of laryngeal cancer is much higher in men than in women, especially for those between 60 and 70 years of age [6]. All patients in the current study were men. Consistent with other studies, we found that patients with total laryngectomy, poor differentiation, T3-T4 stage, N1-N2 stage or III-IV TNM stage had reduced survival [9-12]. We developed multivariate Cox regression analysis models that adjusted for the most important covariates, including SNP, tumor differentiation, neck dissection, $\mathrm{T}$ stage and TNM stage. In some researches indicated that patients T-stage and $\mathrm{N}$-stage had demonstrated to be significantly associated with survival and these findings are consistent with results of most other studies. Our study also obtained the consistent conclusion $[9,13,14]$. A multivariate analysis was conducted, taking the variables found to be significant in univariate analyses $(p \leq 0.2)$ into account. We showed that rs 11903757 and rs966423 polymorphisms correlate with LSCC patient survival. In particular, theS rs 11903757 CT and rs 966423
TT genotypes correlated with poorer outcomes in this patient group.

Rs11903757 is an intergenic SNP on chromosome $2 \mathrm{q} 32.3$ with closest proximity to $N A B P 1$ (44 kb centromeric) and $S D P R$ (112 kb telomeric), which encodes the serum-deprivation response phosphatidylserinebinding protein [15]. The CT genotype was associated with reduced survival. Peters, et al. [16] recently reported a genome-wide association between rs11903757 and colorectal cancer risk in a combined analysis of European and Asian case-control studies. However, another study [17] failed to corroborate previously published data showing an association between rs11903757 and colorectal cancer risk.

Rs966423 is located in the DIRC3 gene intron region at $2 \mathrm{q} 35$ and the TT genotypes were associated with worse survival in SCLC patients. DIRC3 (disrupted in renal cancer 3 ) was first identified in 2003. Its disruption by a $t(2 ; 3)$ $(\mathrm{q} 35 ; \mathrm{q} 21)$ translocation was observed in renal cell carcinoma [18], and although the function of DIRC3 is unknown, it is presumed to have tumor suppressor activity. In a genomewide association study, DIRC3 was associated both with thyroid cancer risk and thyroid stimulating hormone level [19]. It is thus possible that DIRC3 changes alter thyroid stimulating hormone production and, indirectly, promote thyroid cancer development as a result of decreased thyroid epithelium differentiation. Additional research is needed to determine whether or not a similar pathway promotes LSCC. The rs966423 TT genotype was reportedly associated with increased overall mortality in patients with differentiated thyroid cancer [20]. The rs966423 [20]. The CT and CT + TT genotypes were more common in papillary thyroid cancer patients with extra-thyroidal extension and more advanced T stage [21-23]. Laryngeal and thyroid carcinoma are the 
two main malignant tumor types of the head and neck, and both sides of the thyroid gland attach to the lower part of the throat. Thyroid carcinomas were incidentally found in $0.7-$ $3 \%$ of surgeries for another primary head and neck cancer of non-thyroid origin [24-29]. A clinically unexpected, simultaneous thyroid cancer confirmed postoperatively from thyroid tissue partially removed with a laryngeal cancer specimen is rare [30], and these findings require additional confirmation.

Our research had some limitations. First, our sample size of 170 patients was small, and a wider sample range is needed to verify our results. Second, our samples were geographically limited to Shaanxi, China, and a larger number of samples from different ethnic populations must be studied. In addition, our study did not elucidate the functional relevance of the variants to gain insight into the mechanisms underlying the association.

In conclusion, this study showed that LSCC patients with total laryngectomy, poor differentiation, T3-T4 stage, N1-N2 stage or III-IV TNM stage had reduced survival and was the first to demonstrate that the rs11903757 GT and rs966423 TT genotypes predict poor patient outcome. These polymorphisms may serve as useful clinical markers to predict patient survival, and to guide individual patient therapeutic decisions.

\section{MATERIALS AND METHODS}

\section{Ethics approval and patient consent}

Informed consent was obtained from each patient according to protocols approved by the ethics committees of the First Affiliated Hospital of Xi'an Jiaotong University School of Medicine.

\section{Subjects}

We identified 170 patients without distant LSCC metastasis who underwent partial or total laryngectomy. Patient blood samples were randomly collected at the First Affiliated Hospital of Xi'an Jiaotong University School of Medicine from January 2002 to April 2013. Histologic tumor diagnosis was made and agreed upon by at least two senior pathologists at the Department of Pathology based on World Health Organization (WHO) criteria. All patients were men aged 32 to 82 years with an average age of 60.75 years. X-rays, computed tomography (CT) scans, laryngoscopy, examination of laryngeal lesions, local cell smears and pathology examinations were used for diagnosis. Eligible patients had pathologically-confirmed laryngeal carcinoma without distant metastases (M0). All patients underwent a standard clinical examination within two months of diagnosis and none had received any therapy before admission for surgery. All cases were systematically classified based on the Union of International Cancer Control (UICC, 2010) TNM staging system of laryngeal carcinomas, which essentially establishes the modality of therapy.
Follow-up included access to medical records and telephone contact. Patient medical records were reviewed to assess patient characteristics, including age, laryngectomy (partial or total), neck dissection (yes or no), tumor differentiation, T-stage, N-stage, TNM stage and final status on the last follow-up examination. We chose April 2013 as the eligibility end time with the goal of having adequate follow-up for individual participants, with a median follow-up time of 48 months (range, 3-122 months). 100 patients died during the study period and no patient was lost from follow-up data. Survival time was defined as from the date of surgery to the date of death.

\section{SNP selection and genotyping}

We selected 14 total SNPs in chromosomes 1, 2 and 3 that had minor allele frequencies (MAF) $>5 \%$ and were associated with cancer of the aerodigestive tract in the HapMap Asian population. We extracted genomic DNA from peripheral blood using a GoldMag-Mini Whole Blood Genomic DNA Purification Kit (GoldMag Ltd. Xi'an, China) according the manufacturer's protocol. SNP genotypes were obtained according to the standard protocol recommended by Sequenom MassARRAY RS1000 [31]. Finally, Sequenom Typer 4.0 Software was used for data management and analysis [31, 32].

\section{Statistical analysis}

Correlations between categoric variables were assessed using the chi-square test. Survival curves were drawn using the Kaplan-Meier method. Differences between curves were analyzed using the log-rank test. A Cox proportional hazards model was applied to estimate risk by calculating hazard ratios (HR) and 95\% confidence intervals (CI) for categorical variables of exposure. Multivariate Cox regression analysis models were then developed that adjusted for the most important covariates. The SPSS statistical software package version 17.0 (SPSS Inc., Chicago, IL, USA) was used for all analyses. $P<0.05$ was considered statistically significant.

\section{ACKNOWLEDGMENTS}

We are grateful to all the patients and individuals for their participation.

\section{CONFLICTS OF INTEREST}

The authors declare no conflicts of interest.

\section{GRANT SUPPORT}

This work is supported by the China Postdoctoral Science Foundation (NO. 2015M572575) and the Key Science and Technology Program of Shaanxi Province, China (NO.2014K11-01-01-09). 


\section{REFERENCES}

1. Jemal A, Siegel R, Ward E, Hao Y, Xu J, Thun MJ. Cancer statistics, 2009. Ca A Cancer Journal for Clinicians. 2009; 59:225-249.

2. Vokes EE, Stenson KM. Therapeutic options for laryngeal cancer. New England Journal of Medicine. 2003; 349:2087-2089.

3. Morshed K. Association between human papillomavirus infection and laryngeal squamous cell carcinoma. Journal of Medical Virology. 2010; 82:1017-1023.

4. Hatakeyama M, Higashi H. Helicobacter pylori CagA: a new paradigm for bacterial carcinogenesis. Cancer Science. 2005; 96:835-843.

5. Zhuo XL, Yan W, Zhuo WL, Zhang XY. Possible association of Helicobacter pylori infection with laryngeal cancer risk: an evidence-based meta-analysis. Archives of Medical Research. 2008; 39:625-628.

6. Jacques F, Hai-Rim S, Freddie B, David F, Colin M, Donald Maxwell P. Estimates of worldwide burden of cancer in 2008: GLOBOCAN 2008. International Journal of Cancer Journal International Du Cancer. 2010; 127:2893-2917.

7. Holgersson G, Ekman S, Reizenstein J, Bergqvist M, Pontén F, Uhlén M, Magnusson K, Jonnalagadda P, Asplund A, Strömberg S. Molecular profiling using tissue microarrays as a tool to identify predictive biomarkers in laryngeal cancer treated with radiotherapy. Cancer Genomics \& Proteomics. 2010; 7:1-7.

8. Zou J, Yang H, Chen F, Zhao H, Lin P, Zhang J, Ye H, Wang L, Liu S. Prognostic significance of fascin-1 and E-cadherin expression in laryngeal squamous cell carcinoma. European Journal of Cancer Prevention. 2010; 19:11-17.

9. Novin K, Ameri A, Faraji S, Torbati P, Mortazavi N. Head and Neck Squamous Cell Carcinoma in Iran: ClinicoPathological and Treatment-Related Factors Influencing Survival. Iranian Journal of Cancer Prevention. 2015; 8.

10. Christophe LT, Guy-Michel J, Christian B, Guy B, Henri F, Michel V. Prognostic factors of survival in head and neck cancer patients treated with surgery and postoperative radiation therapy. Acta oto-laryngologica. 2008; 128:706-712.

11. Kowit P, Sumet P, Vitoon L, Wattana S, Jaturong J, Temsak $\mathrm{P}$, Kanyarat C, Paramee T. Survival and prognostic factors of different sites of head and neck cancer: an analysis from Thailand. Asian Pacific Journal of Cancer Prevention Apjcp. 2012; 13:885-890.

12. Bing-Hui Z, Wei L, Liang L, Jian-Guang L, Ya-Nan S, De-Jun J, Xiu-Yu X. KAI1/CD82 and MRP1/CD9 serve as markers of infiltration, metastasis, and prognosis in laryngeal squamous cell carcinomas. Asian Pacific Journal of Cancer Prevention Apjcp. 2013; 14:3521-3526.

13. Pruegsanusak K, Peeravut S, Leelamanit V, Sinkijcharoenchai W, Jongsatitpaiboon J, Phungrassami T, Chuchart K, Thongsuksai P. Survival and prognostic factors of different sites of head and neck cancer: an analysis from Thailand. Asian Pacific journal of cancer prevention: APJCP. 2012; 13:885-890

14. Thompson TL, Pagedar NA, Karnell LH, Funk GF. Factors associated with mortality in 2-year survivors of head and neck cancer. Archives of otolaryngology-head \& neck surgery. 2011; 137:1100-1105.

15. Ulrike P, Shuo J, Schumacher FR, Hutter CM, Aragaki AK, Baron JA, Berndt SI, Stéphane B, Hermann B, Katja B. Identification of Genetic Susceptibility Loci for Colorectal Tumors in a Genome-Wide Meta-analysis. Gastroenterology. 2013; 144:799-807.

16. Peters U, Jiao S, Schumacher FR, Hutter CM, Aragaki AK, Baron JA, Berndt SI, Bézieau S, Brenner H, Butterbach K. Identification of Genetic Susceptibility Loci for Colorectal Tumors in a Genome-Wide Meta-analysis. Gastroenterology. 2013; 144:799-807.

17. Nicola W, Hosking FJ, Farrington SM, Claire P, Dobbins SE, Lina Z, Amy L, Ben K, Maggie G, Albert T. Identification of susceptibility loci for colorectal cancer in a genome-wide meta-analysis. Human Molecular Genetics. 2014; 23:4729-4737.

18. Dani?Lle B, Marga S, Eleveld MJ, Schoenmakers EFPM, Ad GVK. Disruption of a novel gene, DIRC3, and expression of DIRC3-HSPBAP1 fusion transcripts in a case of familial renal cell cancer and $\mathrm{t}(2 ; 3)(\mathrm{q} 35 ; \mathrm{q} 21)$. Genes Chromosomes \& Cancer. 2003; 38:107-116.

19. Julius G, Patrick S, Gudbjartsson DF, Jonasson JG, Gisli M, Huiling H, Aslaug J, Asgeir S, Stacey SN, Hrefna J. Discovery of common variants associated with low TSH levels and thyroid cancer risk. Nature Genetics. 2012; 44.

20. Swierniak M, Wojcicka A, Czetwertynska M, Długosinska J, Stachlewska E, Gierlikowski W, Kot A, Gornicka B, Koperski L, Bogdanska M. Association Between GWASderived rs966423 Genetic Variant and Overall Mortality in Patients with Differentiated Thyroid Cancer. Clinical Cancer Research. 2015.

21. Wei WJ, Lu ZW, Yu W, Zhu YX, Wang YL, Ji QH. Clinical significance of papillary thyroid cancer risk loci identified by genome-wide association studies. Cancer Genetics. 2015; 208:68-75.

22. Wang Y-L, Feng S-H, Guo S-C, Wei W-J, Li D-S, Wang Y, Wang X, Wang Z-Y, Ma Y-Y, Jin L. Confirmation of papillary thyroid cancer susceptibility loci identified by genome-wide association studies of chromosomes 14q13, $9 q 22,2 q 35$ and 8 p12 in a Chinese population. Journal of medical genetics. 2013; 50:689-695.

23. Sandya Liyanarachchi AW, Wei Li, Malgorzata Czetwertynska, Elzbieta Stachlewska, Rebecca Nagy, Kevin Hoag, Bernard Wen, Rafal Ploski, Matthew D. Ringel, Izabella Kozłowicz-Gudzinska, Wojciech Gierlikowski, Krystian Jazdzewski, Huiling He, Albert de la Chapelle. Cumulative Risk Impact of Five Genetic Variants Associated With Papillary Thyroid Carcinoma. Thyroid. 2013; 23:1532-1540. 
24. Marco G, Pasquale Q, Ettore S, Roberto B, Franco M. Thyroid carcinoma associated with squamous cell carcinoma of the head and neck: which policy? Head \& Neck. 2007; 29:33\&ndash;37.

25. Pacheco-Ojeda L, Micheau C, Luboinski B, Richard J, Travagli JP, Schwaab G, and Marandas P, Squamous Cell Carcinoma of the Upper Aerodigestive Tract Associated With Well-Differentiated Carcinoma of the Thyroid Gland. Laryngoscope. 1991; 101:421-424.

26. Farrag TY, Lin FC, Sciubba JJ, Koch WM, Flint PW, Tufano RP. Importance of routine evaluation of the thyroid gland prior to open partial laryngectomy. Archives of Otolaryngology-Head \& Neck Surgery. 2006; 132:1047-1051.

27. Pitman KT, Johnson JT, Myers EN. Papillary thyroid carcinoma associated with squamous cell carcinoma of the head and neck: significance and treatment. American Journal of Otolaryngology. 1996; 17:190-196.

28. Coskun H, Erisen L, Tolunay S, Basut O, Onart S, Tezel I. Incidental association of thyroid carcinoma and squamous cell carcinoma of head and neck. American Journal of Otolaryngology. 2002; 23:228-232.

29. Fliegelman L, Genden E, Brandwein M, Mechanick J, Urken M. Significance and management of thyroid lesions in lymph nodes as an incidental finding during neck dissection. Head \& Neck. 2001; 23:885-891.

30. Shih C, Wang CP, Lou PJ, Hu YL, Yang TL, Ko JY, Lee SY. Thyroid cancer incidentally found in radical surgery for laryngeal/hypopharyngeal cancer. Otolaryngology-head and neck surgery: official journal of American Academy of Otolaryngology-Head and Neck Surgery. 2009; 141:343-346.

31. Gabriel S, Ziaugra L, Tabbaa D. SNP genotyping using the Sequenom MassARRAY iPLEX platform. Current protocols in human genetics/editorial board, Jonathan L Haines [et al]. 2009; Chapter 2:2.12.11-12.12.16.

32. Thomas RK, Baker AC, Debiasi RM, Winckler W, Laframboise T, Lin WM, Wang M, Feng W, Zander T, Macconaill LE. High-throughput oncogene mutation profiling in human cancer. Nature Genetics. 2007; 39:347-351. 